
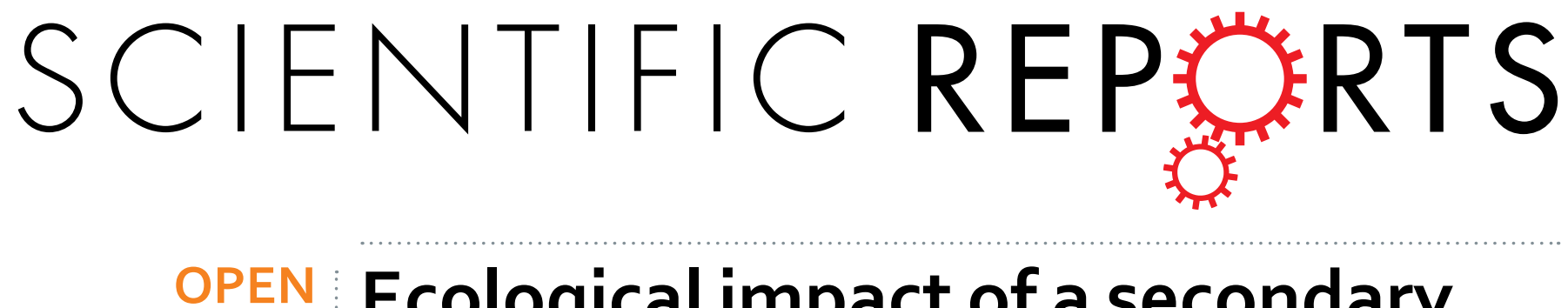

\title{
Ecological impact of a secondary bacterial symbiont on the clones of Sitobion avenae (Fabricius) \\ (Hemiptera: Aphididae)
}

Received: 14 July 2016

Accepted: 09 December 2016

Published: 17 January 2017

\author{
Chen $\mathrm{Luo}^{1,2, *}$, Kun Luo ${ }^{1, *}$, Linqin Meng ${ }^{1}$, Bin Wan², Huiyan Zhao ${ }^{1}$ \& Zuqing Hu ${ }^{1}$
}

Many insects harbor heritable endosymbionts, whether obligatory or facultative, and the role of facultative endosymbionts in shaping the phenotype of these species has become increasingly important. However, little is known about whether micro-injected endosymbionts can have any effects on aphid clones, which was measured using various ecological parameters. We examined the effects between symbiotic treatments and the vital life history traits generated by Regiella insecticola on the life table parameters of Sitobion avenae. The results showed that $R$. insecticola can decrease the intrinsic rate of increase $(r)$, the finite rate of increase $(\lambda)$ and birth rate and can increase the mean generation times $(T)$ of $S$. avenae clones, suggesting that $R$. insecticola may decelerate the normal development of the hosts. No significant differences of these parameters were observed between the examined Sitobion avenae clones, and the symbiont treatment by genotype interaction affected only the net reproduction rate $R_{0}$, pre-adult duration and total longevity but not the other parameters. Additionally, a population projection showed that $R$. insecticola decelerated the growth of the $S$. avenae clones. The evocable effects of $R$. insecticola on the $S$. avenae clones may have significant ramifications for the control of S. avenae populations under field/natural conditions.

Many insect species harbor endosymbionts, which are indispensable to their survival and common reproduction. Because the hosts and their endosymbionts share a common fate, endosymbionts can often profoundly affect the ecology and evolution of their hosts. Among them, the endosymbionts in aphids are ideal targets for studying their role in ecological fitness ${ }^{1}$. In nature, almost all aphid species possess the primary endosymbiont Buchnera aphidicola, which belongs to the class $\gamma$-proteobacterium. B. aphidicola synthesizes essential amino acids and other nutrients that are ingested in very small quantities from restricted diets, such as plant phloem, for their host aphids ${ }^{2-4}$. Moreover, aphids can also be infected with one or more non-essential secondary endosymbionts belonging to distinct bacterial lineages ${ }^{1}$. For example, Regiella insecticola, Hamiltonella defensa and Serratia symbiotica belong to the class $\gamma$-proteobacterium, and Rickettsia and Spiroplasma belong to the class $\alpha$-proteobacterium ${ }^{5-13}$.

Secondary endosymbionts do not present in all aphid individuals, suggesting they are not generally required for the survival and reproduction of aphids. However, endosymbionts can significantly affect host aphid ecological fitness and behavior. In the past few decades, secondary endosymbionts have been found to confer benefits on the pea aphid Acyrthosiphon pisum (Harris), including resistance to heat shock (S. symbiotica ${ }^{14}$ ), protection against natural enemies ( $H$. defensa and $S$. symbiotica ${ }^{15}, H$. defens $a^{16}, R$. insecticola ${ }^{17}$, Spiroplasma ${ }^{18}$ and Rickettsiella $\left.{ }^{19}\right)$, adjustment to reproduction $\left(\right.$ Spiroplasma $\left.^{20}\right)$, host utilization $\left(R\right.$. insecticola $\left.{ }^{21}\right)$, wing and sexual induction $\left(R\right.$. insecticola ${ }^{22}$ ) and body color (Rickettsiella $\left.{ }^{23}\right)$. To date, there is evidence that secondary endosymbionts play major roles in protecting host aphids against natural enemies ${ }^{17,24}$, and their effects on host aphid population dynamics ${ }^{25}$ can affect the communities involved ${ }^{26}$. To examine the dynamic population parameters affected by endosymbionts, an appropriate study of the life history of aphids is essential. Wang et al. ${ }^{27}$ reported

${ }^{1}$ State Key Laboratory of Crop Stress Biology for Arid Areas, College of Plant Protection, Northwest A\&F University, Yangling, Shaanxi Province 712100, China. ${ }^{2}$ INRA (French National Institute for Agricultural Research), Univ. Nice Sophia Antipolis, CNRS, UMR 1355-7254 Institut Sophia Agrobiotech, 06903, Sophia Antipolis, France. ${ }^{*}$ These authors contributed equally to this work. Correspondence and requests for materials should be addressed to Z.H. (email: huzuqing@nwsuaf.edu.cn) 


\begin{tabular}{|c|c|c|c|c|c|c|c|c|c|c|c|c|c|c|c|c|c|c|c|c|}
\hline & \multicolumn{5}{|c|}{ Intrinsic rate of increase $r$} & \multicolumn{5}{|c|}{ Mean generation times $T$} & \multicolumn{5}{|c|}{ Finite rate of increase $\lambda$} & \multicolumn{5}{|c|}{ Net reproductive rate $R_{0}$} \\
\hline & SS & df & MS & $F$ & $P$ & SS & df & MS & $F$ & $P$ & SS & df & MS & $F$ & $P$ & ss & df & MS & $F$ & $P$ \\
\hline R. insecticola & 0.010 & 2 & 0.005 & 38.448 & $<0.001$ & 15.321 & 2 & 7.661 & 22.527 & $<0.001$ & 0.018 & 2 & 0.009 & 39.547 & $<0.001$ & 109.385 & 2 & 54.693 & 9.246 & 0.004 \\
\hline Clone (R. insecticola) & 0.001 & 3 & $<0.001$ & 2.727 & 0.091 & 1.656 & 3 & 0.552 & 1.623 & 0.236 & 0.002 & 3 & 0.001 & 2.730 & 0.090 & 212.312 & 3 & 70.771 & 11.965 & 0.001 \\
\hline Error & 0.002 & 12 & $<0.001$ & - & - & 4.081 & 12 & 0.340 & - & - & 0.003 & 12 & $<0.001$ & - & - & 70.980 & 12 & 5.915 & - & - \\
\hline
\end{tabular}

Table 1. Estimates of variance components for population parameters (i.e., the intrinsic rate of increase $r$, the mean generation times $T$, the finite rate of increase $\lambda$ and the net reproductive rate $R_{0}$ ) of Sitobion avenae clones showing the main effects of Regiella insecticola and clone (i.e., 'Linyi' and 'Neixiang') nested in the Regiella insecticola. A linear two-way nested analysis of variance (ANOVA) was performed. Bold text indicates significant effects.

the life history of R. insecticola in S. avenae using antibiotics to selectively cure infected lines (the elimination of secondary symbionts using $100 \mu \mathrm{g} \mathrm{ml}^{-1}$ ampicillin, $50 \mu \mathrm{g} \mathrm{ml}^{-1}$ cefotaxime and $50 \mu \mathrm{g} \mathrm{ml}^{-1}$ gentomicin). However, a key component of this knowledge is the life table. The life table analysis is the most reliable method for evaluating the survival and reproduction of a population ${ }^{28}$, and some vital ecological parameters are derived from a life table analysis. For instance, the intrinsic rate of increase $(r)$ represents the reproductive potential of a population in relation to its capacity to increase ${ }^{29}$; the finite rate of increase $(\lambda)$ was defined as the rate of increase per individual per unit time; and the net reproductive rate $\left(R_{0}\right)$ has been used to summarize the mean number of offspring that an individual can produce during its lifetime, with the mean generation time $(T)$ defined as the period that a population needs to increase its size $R_{0}$-fold ${ }^{30}$. At the same time, the inoculation (by injection or feeding) and elimination of secondary symbionts are complementary methods. It is often best to use both methods to demonstrate an effect of these bacteria, and adding and removing bacteria can present the predicted phenotypic effects. However, elimination of secondary symbionts with antibiotics may generate the concurrent harm to the primary symbiont, Buchnera, on which aphid performance and fecundity depends ${ }^{15}$. Therefore, for a more exact understanding of the impacts of $R$. insecticola on hosts, it is necessary to obtain $S$. avenae clones with $R$. insecticola inoculated via micro-injection, which would have the same genetic background as symbiont-free aphids. Additionally, different aphid species or different genetic backgrounds within one species in the field can harbor various endosymbionts, and it is not clear whether the genotypes of the hosts could impact the ecological effects of endosymbionts. Therefore, the study of the interactive effects between micro-injected endosymbionts and the genotypes of the hosts and the ecological effects of endosymbionts on the aphid population is warranted.

The objectives of the study are to investigate the ecological effects of $R$. insecticola on different $S$. avenae clones based on the life table data. The results that we present here might provide new insight for the future systematic study of endosymbionts and its ramifications in suppressing $S$. avenae populations.

\section{Results}

The effects of $R$. insecticola on population parameters in different aphid genotypes (clones). A two-way nested analysis of variance was used to analyze the effect of endosymbionts on the population parameters in the different genotypes of aphid host (Table 1 ). $R$. insecticola significantly impacted the population parameters (i.e., $P<0.001$ for the intrinsic rate of increase $r$, mean generation times $T$, finite rate of increase $\lambda$ and net reproductive rate $\left.R_{0}\right)$. Clone nested in ' $R$. insecticola' had no impact on $r(P=0.091), T(P=0.236)$ and $\lambda$ $(P=0.090)$, but significantly impacted $R_{0}(P=0.001)$.

The population parameters of these original aphid clones (controls) were compared to those corresponding to infected clones (treatments) (Table 2). Body fluids from the Yangpingguan clones 1 and 2 were transferred to the Linyi clone and the Neixiang clone, respectively, resulting in the creation of four new clones (Linyi-1 and Neixiang-1 from Yangpingguan clone 1 donor, Linyi-2 and Neixiang-2 from Yangpingguan clone 2 donor). The four clones presented significantly lower values $(F=27.418, P<0.001$ and $F=28.216, P<0.001$ for $r$ and $\lambda$, respectively) than the controls Linyi-NA (Linyi clone) and Neixiang-NA (Neixiang clone). There were no differences between the treatments (Linyi-1, Linyi-2, Neixiang- 1 and Neixiang-2) $(P=0.191$ and $P=0.194$ for $r$ and $\lambda$, respectively). However, the mean generation times $(T)$ of treatments Linyi-1, Linyi-2, Neixiang-1 and Neixiang-2 were significantly longer $(F=19.553, P<0.001)$ than that of the controls Linyi-NA and Neixiang-NA, and no differences were found between the treatments (Linyi-1, Linyi-2, Neixiang-1 and Neixiang-2) $(P=0.119)$.

The effects of $R$. insecticola on the development and longevity of different $S$. avenae genotypes. A two-way nested analysis of variance was used to analyze the effect of endosymbionts on fitness traits in the different genotypes of the aphid host (Table 3). R. insecticola clearly affected the birth rate $(P<0.001)$, pre-adult duration $(P<0.001)$ and total longevity $(P=0.001)$. Clone nested in ' $R$. insecticola' had no impact on birth rate $(P=0.091)$ but had a significant impact on pre-adult duration and total longevity $(P=0.018$ and $P=0.004$, respectively).

Fitness traits (i.e., birth rates, pre-adult duration, and total longevity) of the controls clones were compared to those corresponding to infected clones (treatments) (Table 4). R. insecticola had a remarkable effect on birth rate, and birth rates in the treatments Linyi-1, Linyi-2, Neixiang-1 and Neixiang-2 were significantly lower $(F=30.404, P<0.001)$ than that in the controls Linyi-NA and Neixiang-NA. No differences in birth rate were found between the treatments (Linyi-1, Linyi-2, Neixiang-1 and Neixiang-2) $(P=0.163)$. The pre-adult duration of the treatments Linyi-1, Linyi-2, Neixiang-1 and Neixiang-2 $(8.32 \pm 0.062,7.51 \pm 0.156,7.80 \pm 0.102$ and $7.97 \pm 0.259$, respectively) were clearly longer than that of the controls Linyi-NA $(7.03 \pm 0.126)$ and Neixiang-NA 


\begin{tabular}{|c|c|c|c|c|c|c|}
\hline \multirow[b]{2}{*}{ Clones } & \multirow[b]{2}{*}{ Genotype } & \multirow[b]{2}{*}{$\begin{array}{l}\text { R. insecticola } \\
\text { donor }\end{array}$} & \multicolumn{4}{|c|}{ Population parameter $($ Mean \pm SE) } \\
\hline & & & $\begin{array}{l}\text { Intrinsic rate of } \\
\text { increase } r\left(d^{-1}\right)\end{array}$ & $\begin{array}{l}\text { Net reproductive rate } \\
R_{0} \text { (offspring/aphid) }\end{array}$ & $\begin{array}{l}\text { Mean generation } \\
\text { times } T(\mathrm{~d})\end{array}$ & $\begin{array}{c}\text { Finite rate of } \\
\text { increase } \lambda\left(d^{-1}\right)\end{array}$ \\
\hline Linyi-1 & Linyi & Yangpingguan 1 & $0.24 \pm 0.003 \mathrm{~b}$ & $38.04 \pm 1.333 \mathrm{a}$ & $14.97 \pm 0.287 \mathrm{a}$ & $1.28 \pm 0.004 \mathrm{~b}$ \\
\hline Linyi-2 & Linyi & Yangpingguan 2 & $0.28 \pm 0.010 \mathrm{~b}$ & $42.50 \pm 0.896 \mathrm{a}$ & $13.64 \pm 0.472 \mathrm{a}$ & $1.32 \pm 0.013 \mathrm{~b}$ \\
\hline Linyi-NA & Linyi & control & $0.30 \pm 0.003 \mathrm{a}$ & $39.14 \pm 2.622 \mathrm{a}$ & $12.06 \pm 0.260 \mathrm{~b}$ & $1.36 \pm 0.004 \mathrm{a}$ \\
\hline Neixiang-1 & Neixiang & Yangpingguan 1 & $0.26 \pm 0.009 \mathrm{~b}$ & $38.20 \pm 0.636 \mathrm{a}$ & $13.91 \pm 0.430 \mathrm{a}$ & $1.30 \pm 0.012 \mathrm{~b}$ \\
\hline Neixiang-2 & Neixiang & Yangpingguan 2 & $0.26 \pm 0.007 \mathrm{~b}$ & $34.43 \pm 0.667 \mathrm{a}$ & $13.66 \pm 0.340 \mathrm{a}$ & $1.30 \pm 0.009 \mathrm{~b}$ \\
\hline Neixiang-NA & Neixiang & control & $0.31 \pm 0.000 \mathrm{a}$ & $47.89 \pm 1.234 \mathrm{a}$ & $12.33 \pm 0.084 b$ & $1.37 \pm 0.000 \mathrm{a}$ \\
\hline
\end{tabular}

Table 2. The effects of $R$. insecticola on the intrinsic rate of increase $r$, the net reproductive rate $R_{0}$, the mean generation times $T$, and the finite rate of increase $\lambda$ of the studied $S$. avenae clones (Linyi-1, Linyi-2, Neixiang-1, Neixiang-2, Linyi-NA and Neixiang-NA) (Mean \pm SE). Different letters (a, b) within a column indicate statistically significant differences $(P<0.05$; Tukey's test was used for mean separation).

$(6.62 \pm 0.950)$. In addition, the total longevity of the treatments Linyi-1, Linyi-2, Neixiang-1 and Neixiang-2 $(27.34 \pm 0.484,27.62 \pm 0.624,26.31 \pm 0.791$ and $25.41 \pm 0.423$, respectively) were clearly higher than that of the control Linyi-NA $(22.29 \pm 0.756)$.

Population projection. The simulated population growth and stage structure of the adult stages for 30 days, beginning with an initial population of 10 pairs of $1^{\text {st }}$ instar nymphs, are shown in Fig. 1 . For the adults, after an early stable phase, the times in which the populations entered sustained population growth (outbreak times) for the treatments Linyi-1, Linyi-2, Neixiang- 1 and Neixiang- 2 occurred on the $17^{\text {th }}, 15^{\text {th }}, 16^{\text {th }}$ and $16^{\text {th }}$ day, respectively, and the corresponding controls Linyi-NA and Neixiang-NA both occurred on the $14^{\text {th }}$ day. This showed that the outbreak times of the treatments were delayed compared to those of the controls, and it suggested that $R$. insecticola might postpone the timing of the adult populations in the studied $S$. avenae clones.

\section{Discussion}

The diverse infections and functions of secondary endosymbionts of aphids (e.g., A. pisum, Aphis craccivora and Myzus persicae) have been extensively studied in recent decades ${ }^{5-9,18,31-36}$. In addition, it has become clear that secondary endosymbionts have major effects on the ecology of the pea aphid and arthropods in general. For example, $H$. defensa can protect $A$. pisum from its natural enemies ${ }^{1,16,18,24}$. However, Łukasik et al. ${ }^{31}$ reported that $H$. defensa has little effect on the resistance to the natural enemies in S. avenae, indicating that secondary endosymbionts might, to some extent, have different effects on related species. Therefore, it is necessary to study the effects of the secondary symbionts on different aphid clones using the life table.

A linear two-way nested analysis of variance (ANOVA) showed that the treatment by genotype interaction did not affect aphid fitness (e.g., $r, T, \lambda$ and birth rate), though the interaction did affect the net reproductive rate $\left(R_{0}\right)$, pre-adult duration and total longevity. For a comprehensive understanding of the treatment by genotype interaction, more studies are needed.

Understanding the entire life history plays a significant role in evaluating the population parameters that are influenced by secondary endosymbionts, and the key component of this analysis is the life table, which can provide a comprehensive description of the development, survival, and fecundity of a population ${ }^{28}$. Wang et al. ${ }^{27}$ obtained the "curing of $R$. insecticola infected lines" through an oral administration of antibiotics, and they studied the change in the developmental time of $1^{\text {st }}$ to $4^{\text {th }}$ instar nymphs (DT1-DT4), the total developmental time of nymphs (DT5), and $10 \mathrm{~d}$ fecundity. However, their research did not focus on the population parameters. In life table research, the population parameters represent the intrinsic rate of increase $r$, the net reproductive rate $R_{0}$, the mean generation times $T$ and the finite rate of increase $\lambda$. In our study, the main life table parameters researched were the population parameters.

The intrinsic rate of increase $(r)$ of the treatments was significantly lower than that of the controls. The intrinsic rate of increase is a key demographic parameter that has been used to summarize the reproductive potential of an animal population in relation to its capacity to increase ${ }^{29}$. Southwood ${ }^{37}$ stated that the intrinsic rate of increase is the most useful life table parameter for comparing the population growth potential under specific climatic and food conditions. Therefore, the negative effect of $R$. insecticola on the potential growth of S. avenae clones is intriguing. This notion was confirmed by the mean generation times $(T)$ of the treatments, which were shorter than that of the controls. Oliver et al. ${ }^{25}$ reported that $H$. defensa shortened the mean generation time $(T)$ of $A$. pisum. Although $R$. insecticola might decelerate the potential growth of $S$. avenae populations, the finite rates of increase $(\lambda)$ were all over 1 between the treatments and the controls, indicating that the studied S. avenae population increased continuously. The net reproductive rates $\left(R_{0}>1\right)$ between the treatments and the controls also confirmed this observation. However, the finite rates of increase $(\lambda)$ of the treatments with the secondary symbionts were remarkably less than that of the controls, suggesting that the daily increase of $S$. avenae clones in the treatment groups was significantly lower than that of the controls. This was confirmed by the birth rates of the treatment groups, which were significantly lower than that of the controls. In conclusion, $R$. insecticola may reduce the potential growth of $S$. avenae clones, and this is consistent with the findings of Wang et al. ${ }^{27}$, suggesting a possibility of a cost action to harbor this secondary endosymbiont. It was expected that the secondary endosymbiont would play a role against the infection of fungal pathogens ${ }^{17}$. 


\begin{tabular}{|c|c|c|c|c|c|c|c|c|c|c|c|c|c|c|c|}
\hline & \multicolumn{5}{|c|}{ Birth rate } & \multicolumn{5}{|c|}{ Pre-adult duration } & \multicolumn{5}{|c|}{ Total longevity } \\
\hline & SS & df & MS & $F$ & $P$ & SS & df & MS & $F$ & $P$ & SS & df & MS & $F$ & $P$ \\
\hline R. insecticola & 0.018 & 2 & 0.009 & 40.872 & $<0.001$ & 4.910 & 2 & 2.455 & 37.663 & $<0.001$ & 29.211 & 2 & 14.605 & 13.200 & 0.001 \\
\hline Clone (R. insecticola) & 0.002 & 3 & 0.001 & 2.721 & 0.091 & 0.974 & 3 & 0.325 & 4.980 & 0.018 & 26.162 & 3 & 8.721 & 7.881 & 0.004 \\
\hline Error & 0.003 & 12 & $<0.001$ & - & - & 0.782 & 12 & 0.065 & - & - & 13.278 & 12 & 1.106 & - & - \\
\hline
\end{tabular}

Table 3. Estimates of variance components for life history traits (i.e., the birth rate, pre-adult duration and total longevity) of Sitobion avenae clones showing the main effects of Regiella insecticola and clone (i.e., 'Linyi' and 'Neixiang') nested in the Regiella insecticola. A linear two-way nested analysis of variance (ANOVA) was performed. Bold text indicates significant effects.

\begin{tabular}{|c|c|c|c|c|c|}
\hline \multirow[b]{2}{*}{ Clones } & \multirow[b]{2}{*}{ Genotype } & \multirow[b]{2}{*}{ R. insecticola donor } & \multicolumn{3}{|c|}{ Life-history traits (Mean \pm SE) } \\
\hline & & & Birth rate & Pre-adult duration (d) & longevity (d) \\
\hline Linyi-1 & Linyi & Yangpingguan 1 & $0.28 \pm 0.004 b$ & $8.32 \pm 0.062 \mathrm{a}$ & $27.34 \pm 0.484 \mathrm{a}$ \\
\hline Linyi-2 & Linyi & Yangpingguan 2 & $0.32 \pm 0.013 \mathrm{~b}$ & $7.51 \pm 0.156 \mathrm{a}$ & $27.62 \pm 0.624 \mathrm{a}$ \\
\hline Linyi-NA & Linyi & control & $0.36 \pm 0.004 \mathrm{a}$ & $7.03 \pm 0.126 b$ & $22.29 \pm 0.756 b$ \\
\hline Neixiang-1 & Neixiang & Yangpingguan 1 & $0.30 \pm 0.011 \mathrm{~b}$ & $7.80 \pm 0.102 \mathrm{a}$ & $26.31 \pm 0.791 \mathrm{a}$ \\
\hline Neixiang-2 & Neixiang & Yangpingguan 2 & $0.30 \pm 0.009 \mathrm{~b}$ & $7.97 \pm 0.259 \mathrm{a}$ & $25.41 \pm 0.423 \mathrm{a}$ \\
\hline Neixiang-NA & Neixiang & control & $0.37 \pm 0.001 \mathrm{a}$ & $6.62 \pm 0.950 \mathrm{~b}$ & $25.68 \pm 0.453 \mathrm{a}$ \\
\hline
\end{tabular}

Table 4. The effects of $R$. insecticola on the birth rate, pre-adult duration and total longevity of the studied S. avenae clones (Linyi-1, Linyi-2, Neixiang-1, Neixiang-2, Linyi-NA and F Neixiang-NA Mean \pm SE).

Compared to the controls, we could conclude that $R$. insecticola increased the time of pre-adult duration. The results are consistent with the report by Wang et al. ${ }^{27}$. In addition, there was the positive effect of the secondary endosymbionts on the longevity of $S$. avenae clones compared to that of the Linyi control. However, there was no effect of the secondary endosymbionts on the clones of Neixiang-1, Neixiang-2 and Neixiang-NA, which may be explained by the difference of geographic populations.

The intrinsic rates of increase $(r)$, finite rates of increase $(\lambda)$, mean generation time $(T)$ and birth rate demonstrated that $R$. insecticola infection had a negative effect on the natural development of $S$. avenae clones, and the impact levels differed among different genetic backgrounds. The difference between genotypes might be due to variations in host nutrients. For example, the effect of nitrogen content and protein quality might affect the fecundity of herbivores ${ }^{38-41}$. Therefore, to accurately and systematically assess the ecological effect of all endosymbionts on aphids, (1) the endosymbiont treatment and genotype interaction should be noted in future studies, (2) all endosymbionts (i.e., primary and secondary), such as S. symbiotica, H. defense and Rickettsia, need to be examined individually, and (3) more donor and recipient clones or species could be used, as only 2 aphid clones were used as donors and 2 aphid clones were recipients in the current study. Such efforts would provide us with a comprehensive perspective that would aid our understanding of the complex symbiotic relationships between aphids and bacterial endosymbionts.

In conclusion, the findings of this study indicate that the interaction effects between $R$. insecticola and the genotypes of $S$. avenae clones did not show any changes in fitness traits, although changes were observed with the net reproductive rate $\left(R_{0}\right)$, pre-adult duration and total longevity. In addition, the significant reduction of fitness traits (e.g., $r, \lambda$ and birth rate) and the clear increase of the mean generation times $(T)$ indicated that $R$. insecticola might have a negative effect on the potential population growth of $S$. avenae.

\section{Materials and Methods \\ Aphid sources. Aphid clones (considered genotypes in this paper) used in this study were established from individuals collected from winter wheat plants on private farm land, with the permission of the owners. Clones without $R$. insecticola were collected in Neixiang $\left(33.03^{\circ} \mathrm{N}, 111.50^{\circ} \mathrm{E}\right)$, Henan Province and in Linyi $\left(35.05^{\circ} \mathrm{N}\right.$, $118.35^{\circ} \mathrm{E}$ ), Shandong Province in May 2014. Collected clones were genotyped at four microsatellite loci (S91, S24, S30, and Sm10) as described in the Supplementary Information (Tables S1 and S2). R. insecticola donor clones were collected in April 2014 in two quadrats, at a distance of $300 \mathrm{~m}$ from each other, in Yangpingguan $\left(34.8^{\circ} \mathrm{N}\right.$, $105.6^{\circ} \mathrm{E}$ ), Shaanxi. They were named Yangpingguan 1 and Yangpingguan 2. Each clone was derived from a sin- gle parthenogenetic $S$. avenae female, and the endosymbionts of the four clones were detected. The primers are shown in the Supplementary Information (Table S3). Clones were laboratory-reared at $20^{\circ} \mathrm{C}$ under a $12 \mathrm{~h} \mathrm{light:}$ $12 \mathrm{~h}$ dark cycle in separate cubical cages (side length $=25 \mathrm{~cm}$ ) on fresh wheat (Triticum aestivum L.) seedlings (variety ' 1376 ') that were changed at ten-day intervals ${ }^{32}$.}

Transfer of $\boldsymbol{R}$. insecticola and further detection. The micro-injection technique advocated by Chen \& Purcell ${ }^{42}$ was used to inoculate $R$. insecticola into either $2^{\text {nd }}$ or $3^{\text {rd }}$ instar S. avenae individuals. To do this, the Yangpingguan clones 1 and 2 (from Shaanxi) that hosted $R$. insecticola were chosen as the donor clones. Body fluids from the Yangpingguan clones 1 and 2 were transferred into the Linyi (from Shandong) and the Neixiang 


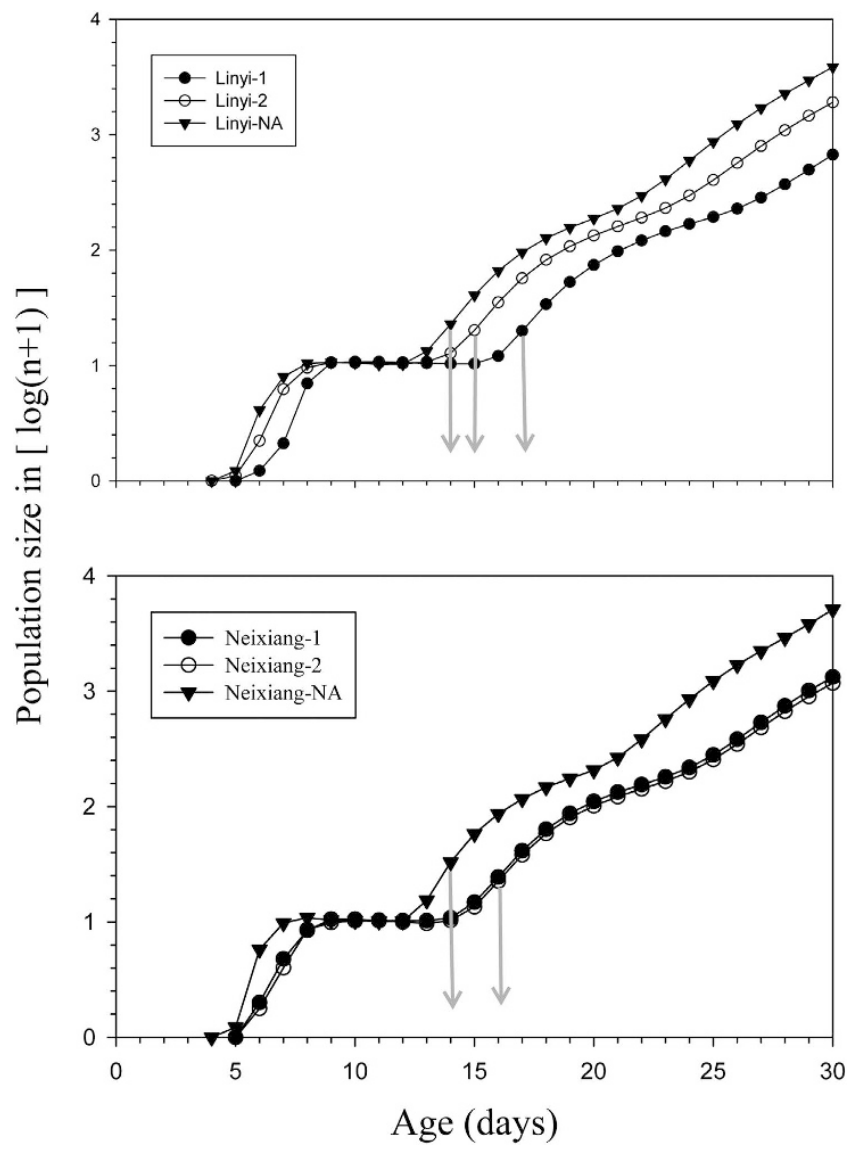

Figure 1. Simulated adult population growth of six S. avenae clones (Linyi-1, Linyi-2, Neixiang-1, Neixiang-2, Linyi-NA and Neixiang-NA) for 30 days. Linyi genotypes with $R$. insecticola (Linyi-1 and Linyi-2) donated by Yangpingguan 1 and Yangpingguan 2, respectively; Neixiang genotypes with $R$. insecticola (Neixiang-1 and Neixiang-2) donated by Yangpingguan 1 and Yangpingguan 2, respectively. Linyi-NA represents Linyi genotype without $R$. insecticola and Neixiang-NA represents Neixiang genotype without $R$. insecticola. The arrow heads point to the times in which the populations entered sustained population growth (outbreak times).

clones (from Henan), respectively, resulting in the creation of four new clones (Linyi-1 and Neixiang-1 from Yangpingguan clone 1 donor, Linyi-2 and Neixiang- 2 from Yangpingguan clone 2 donor). Therefore, the Linyi- 1 and Linyi-2 clones and the Neixiang-1 and Neixiang-2 clones have the same genetic background as the Linyi (Shandong) and the Neixiang clones (Shandong), respectively. The surviving aphids (approximately 20 individuals for clones Linyi-1, Linyi-2, Neixiang-1, and Neixiang-2) after the injection were transferred to fresh wheat plants (one aphid per plant). Twelve hours later, the dead aphids were discarded, and the remaining surviving aphids were reared till they became adults and produce nymphs. Next, one single offspring individual from each clone that tested "positive" was subjected to PCR using PCR primers described in Sandström et al. ${ }^{9}$, Taq DNA polymerase mix, and the following cycle parameters: $94^{\circ} \mathrm{C}$ for $7 \mathrm{~min}$ and 35 cycles of $94^{\circ} \mathrm{C}$ for $30 \mathrm{~s}, 57^{\circ} \mathrm{C}$ for $45 \mathrm{~s}$ and $72^{\circ} \mathrm{C}$ for $30 \mathrm{~s}$. The resulting PCR products were separated in a $1 \%$ agarose gel and visualized under UV after gel staining with ethidium bromide for the presence of $R$. insecticola and were then placed on T. aestivum and allowed to reproduce for several days. At this time, adults were removed and 5 individuals were tested again for the presence of $R$. insecticola. One positive clone was then used to conduct the following study. To ensure that $R$. insecticola densities in $S$. avenae produced clones approached equilibrium, the original data of the life table were collected after a minimum of 10 generations ${ }^{5}$ and statistically analyzed.

Life table study. Linyi-1 and Linyi-2 clones were compared with the Linyi clone control as they all shared a common genetic background. This clone was named Linyi-NA for convenience. Similarly, Neixiang-1 and Neixiang-2 clones were compared with the Neixiang clone control, and the control was named Neixiang-NA for convenience. A preliminary experiment showed that the control clone (no injection) and the negative control (injection of water) showed no difference in the growth and development of the studied S. avenae clones (unpublished data). Experiments were performed using three groups for each individual clone, with a total of 30 aphids in each group. Newly emerged $1^{\text {st }}$ instar nymphs were individually transferred within $24 \mathrm{~h}$ to a 9 -cm Petri dish containing a fresh wheat leaf blade to start the experiment. The collection of life table data was initiated approximately $24 \mathrm{~h}$ later. Each aphid was observed daily at the same time until all individuals died. All offspring were 
removed daily, and the wheat leaves were replaced every 2 days. Molting and mortality events, as well as the number of produced nymphs, were recorded.

Life table analysis. The developmental time and daily fecundity of all individuals were analyzed according to an age-stage, two-sex life table ${ }^{43,44}$ using the program TWOSEXMS Chart ${ }^{45}$. The intrinsic rate of increase $(r)$ was estimated using an interactive bisection method and the following Euler-Lotka equation (equation 1), with the age indexed from $0^{46}$ :

$$
\sum_{x=0}^{\infty} e^{-r(x+1)} l_{x} m_{x}=1
$$

The net reproductive rate $\left(R_{0}\right)$ was calculated as follows (equation 2$)$ :

$$
R_{0}=\sum_{x=0}^{\infty} l_{x} m_{x}
$$

The finite rate of increase $\lambda$ (equation 3) and the mean generation time $T$ (equation 4) were calculated as follows:

$$
\begin{gathered}
\lambda=e^{r} \\
T=\frac{\ln R_{0}}{r}
\end{gathered}
$$

Population projection. Based on the results of the age-stage, two-sex life table, a TIMING-MS Chart was used to predict the population growth of the studied S. avenae clones. For this prediction, we assumed unlimited growth with an initial population of 10 pairs of newly emerged S. avenae nymphs ${ }^{29,47,48}$.

Experimental design and data analysis. A $2 \times 3$ factorial randomized in complete blocks with 30 replications was deployed to test $R$. insecticola on Sitobion avenae clones. All data were analyzed using IBM SPSS version 20 . The differences in life history traits produced from the life table were separately subjected to a linear two-way nested analysis of variance (ANOVA) to analyze the effects of ' $R$. insecticola' and clone (i.e., 'Linyi' and 'Neixiang') nested in ' $R$. insecticola'. We used Tukey's procedure ${ }^{49}$ to compare the differences among treatments, following the description of Sokal \& Rohlf ${ }^{50}$. All mean separation tests were performed at $P<0.05$. Mean values ( \pm standard errors of the mean) were calculated and used in the above analyses. If needed, data were transformed to meet the required assumptions of normality and homoscedasticity. Graphs were generated using SigmaPlot 12.5.

\section{References}

1. Oliver, K. M., Degnan, P. H., Burke, G. R. \& Moran, N. A. Facultative symbionts in aphids and the horizontal transfer of ecologically important traits. Annu. Rev. Entomol. 55, 247-266 (2010).

2. Douglas, A. E. Mycetocyte symbiosis in insects. Biol. Rev. 64, 409-434 (1989).

3. Douglas, A. E. Nutritional interactions in insect-microbial symbioses: aphids and their symbiotic bacteria Buchnera. Annu. Rev. Entomol. 43, 17-37 (1998).

4. Baumann, P. et al. Genetics physiology and evolutionary relationships of the genus Buchnera: intracellular symbionts of aphids. Annu. Rev. Microbiol. 49, 55-94 (1995).

5. Chen, D., Montllor, C. B. \& Purcell, A. H. Fitness effects of two facultative endosymbiotic bacteria on the pea aphid, Acyrthosiphon pisum, and the blue alfalfa aphid. A kondoi. Entomol. Exp. Appl. 95, 315-323 (2000).

6. Darby, A. C., Birkle, L. M., Turner, S. L. \& Douglas, A. E. An aphid-borne bacterium allied to the secondary symbionts of whitefly. FEMS Microbiol. Ecol. 36, 43-50 (2001).

7. Fukatsu, T., Tsuchida, T., Nikoh, N. \& Koga, R. Spiroplasma symbiont of the pea aphid, Acyrthosiphon pisum (Insecta: Homoptera). Appl. Environ. Microb. 67, 1284-1291 (2001).

8. Fukatsu, T., Nikoh, N., Kawai, R. \& Koga, R. The secondary endosymbiotic bacterium of the pea aphid Acyrthosiphon pisum (Insecta: Homoptera). Appl. Environ. Microb. 66, 2748-2758 (2000).

9. Sandström, J. P., Russell, J. A., White, J. P. \& Moran, N. A. Independent origins and horizontal transfer of bacterial symbionts of aphids. Mol. Ecol. 10, 217-228 (2001).

10. Darby, A. C., Chandler, S. M., Welburn, S. C. \& Douglas, A. E. Aphid-symbiotic bacteria cultured in insect cell lines. Appl. Environ. Microb. 71, 4833-4839 (2005).

11. Moran, N. A., Russell, J. A., Koga, R. \& Fukatsu, T. Evolutionary relationships of three new species of Enterobacteriaceae living as symbionts of aphids and other insects. Appl. Environ. Microb. 71, 3302-3310 (2005).

12. Sakurai, M., Koga, R., Tsuchida, T., Meng, X. Y. \& Fukatsu, T. Rickettsia symbiont in the pea aphid Acyrthosiphon pisum: novel cellular tropism, effect on host fitness, and interaction with the essential symbiont Buchnera. Appl. Environ. Microb. 71, 4069-4075 (2005).

13. Tsuchida, T., Koga, R., Meng, X. Y., Matsumoto, T. \& Fukatsu, T. Characterization of a facultative endosymbiotic bacterium of the pea aphid Acyrthosiphon pisum. Microb. Ecol. 49, 126-133 (2005)

14. Montllor, C. B., Maxmen, A. \& Purcell, A. H. Facultative bacterial endosymbionts benefit pea aphids Acyrthosiphon pisum under heat stress. Ecol. Entomol. 27, 189-195 (2002).

15. Oliver, K. M., Russell, J. A., Moran, N. A. \& Hunter, M. S. Facultative bacterial symbionts in aphids confer resistance to parasitic wasps. Proc. Natl. Acad. Sci. USA 100, 1803-1807 (2003).

16. Haine, E. R. Symbiont-mediated protection. Proc. R. Soc. Lond. [Biol]. 75, 353-361 (2008)

17. Scarborough, C. L., Ferrari, J. \& Godfray, H. C. J. Aphid protected from pathogen by endosymbiont. Science. 310, 1781-1781 (2005).

18. Łukasik, P., Asch, M. V., Guo, H., Ferrari, J. \& Godfray, H. C. J. Unrelated facultative endosymbionts protect aphids against a fungal pathogen. Ecol. Lett. 16, 214-218 (2013). 
19. Parker, B. J., Spragg, C. J., Altincicek, B. \& Gerardo, N. M. Symbiont-mediated protection against fungal pathogens in pea aphids: a role for pathogen specificity? Appl. Environ. Microbiol. 79, 2455-2458 (2013).

20. Simon, J. et al. Facultative symbiont infections affect aphid reproduction. PLoS One. 6, e21831 (2011).

21. Tsuchida, T., Koga, R. \& Fukatsu, T. Host plant specialization governed by facultative symbiont. Science. 303, 1989-1989 (2004).

22. Leonardo, T. M. \& Mondor, E. B. Symbiont modifies host life-history traits that affect gene flow. Proc. R. Soc. Lond. [Biol]. 273, 1079-1084 (2006).

23. Tsuchida, T. et al. Symbiotic bacterium modifies aphid body color. Science. 330, 1102-1104 (2010).

24. Brownlie, J. C. \& Johnson, K. N. Symbiont-mediated protection in insect hosts. Trends. Microbiol. 17, 348-354 (2009).

25. Oliver, K. M., Campos, J., Moran, N. A. \& Hunter, M. S. Population dynamics of defensive symbionts in aphids. Proc. R. Soc. Lond. [Biol]. 275, 293-299 (2008).

26. Ferrari, J. \& Vavre, F. Bacterial symbionts in insects or the story of communities affecting communities. Philos. Trans. R. Soc. Lond. B. Biol. Sci. 366, 1389-1400 (2011).

27. Wang, D. et al. Comparison of fitness traits and their plasticity on multiple plants for Sitobion avenae infected and cured of a secondary endosymbiont. Sci. Rep. 6, 23177 (2016).

28. Farhadi, R., Allahyari, H. \& Chi, H. Life table and predation capacity of Hippodamia variegata (Coleoptera: Coccinellidae) feeding on aphis fabae (Hemiptera: Aphididae). Biol. Contr. 59, 83-89 (2011).

29. Andrewartha, H. G. \& Birch, L. C. The distribution and abundance of animals (University of Chicago Press, 1964).

30. Tuan, S., Li, N., Yeh, C., Tang, L. \& Chi, H. Effects of Green manure cover crops on spodoptera litura (Lepidoptera: Noctuidae) populations. J. Econ. Entomol. 107, 897-905 (2014).

31. Łukasik, P., Dawid, M. A., Ferrari, J. \& Godfray, H. C. J. The diversity and fitness effects of infection with facultative endosymbionts in the grain aphid, Sitobion avenae. Oecologia. 173, 985-996 (2013).

32. Luo, C., Luo, K., Hu, Z. Q., Tao, Y. Y. \& Zhao, H. Y. The infection frequencies and dynamics of three secondary endosymbionts in the laboratory environments on Sitobion avenae (Fabricius) as determined by long PCR. J. Asia-Pac. Entomol. 19, 473-476 (2016).

33. Brady, C. M. et al. Worldwide populations of the aphid aphis craccivora are infected with diverse facultative bacterial symbionts. Microb. Ecol. 67, 195-204 (2014).

34. Liu, Y. H. et al. Nitrogen hurdle of host alternation for a polyphagous aphid and the associated changes of endosymbionts. Sci. Rep. 6, 24781 (2016).

35. Asplen, M. K. et al. Specialisation of bacterial endosymbionts that protect aphids from parasitoids. Ecol. Entomol. 39, 736-739 (2014).

36. Vorburger, C. \& Gouskov, A. Only helpful when required: a longevity cost of harbouring defensive symbionts. J. Evolution. Biol. 24, $1611-1617$ (2011).

37. Southwood, T. R. E. Ecological methods, with particular reference to the study of insect populations (Chapman \& Hall, 1966).

38. Awmack, C. S. \& Leather, S. R. Host plant quality and fecundity in herbivorous insects. Annu. Rev. Entomol. 47, 817-844 (2002).

39. Blanco, C. A. et al. Plant host effect on the development of Heliothis virescens F. (Lepidoptera: Noctuidae). Environ. Entomol. 37, $1538-1547$ (2008).

40. Lee, K. P. The interactive effects of protein quality and macronutrient imbalance on nutrient balancing in an insect herbivore. J. Exp. Biol. 210, 3236-3244 (2007).

41. Hwang, S., Liu, C. \& Shen, T. Effects of plant nutrient availability and host plant species on the performance of two Pieris butterflies (Lepidoptera: Pieridae). Biochem. Syst. Ecol. 36, 505-513 (2008).

42. Chen, D. \& Purcell, A. H. Occurrence and transmission of facultative endosymbionts in aphids. Curr. Microbiol. 34, 220-225 (1997).

43. Chi, H. \& Liu, H. Two new methods for the study of insect population ecology. Bull. Inst. Zool. Acad. Sin. 24, 225-240 (1985).

44. Chi, H. Life-table analysis incorporating both sexes and variable development rates among individuals. Environ. Entomol. 17, 26-34 (1988).

45. Chi, H. TWOSEX-MSChart: a computer program for the age-stage, two-sex life table analysis. National Chung Hsing University, Taichung Taiwan. Available at: http://140.120.197.173/Ecology/ (2012).

46. Goodman, D. Optimal life histories, optimal notation, and the value reproductive value. Am. Nat. 803-823 (1982).

47. Chi, $\mathrm{H}$. Timing of control based on the stage structure of pest population: a simulation approach. J. Econ. Entomol. 83, 1143-1150 (1990).

48. Chi, H. Timing-MSChart: a computer program for the population projection based on age-stage, two-sex life table. National Chung Hsing University, Taichung Taiwan. Available at: http://140.120.197.173/Ecology/ (2013).

49. Dunnett, C. W. Pairwise multiple comparisons in the unequal variance case. J Am. Stat. Assoc. 75, 796-800 (1980).

50. Rohlf, F. J. \& Sokal, R. R. Statistical tables (Macmillan, 1995).

\section{Acknowledgements}

The authors are grateful to Marylène Poirié for her critical comments on the logic and sentences of this manuscript and to the anonymous reviewers for their critical comments on earlier versions of this manuscript. This work was supported by the National Natural Science Foundation of China (31401735), the China Postdoctoral Science Foundation funded project (2015T81057, 2014M560807), and project funding by the Postdoctoral Science Foundation of Shaanxi Province.

\section{Author Contributions}

H.Z., Z.H., C.L., and K.L. conceived and designed the experiments. C.L., L.M., and K.L. performed the experiments. C.L. and Z.H. analyzed the data. C.L., Z.H., B.W., and K.L. wrote the paper. All authors reviewed the manuscript.

\section{Additional Information}

Supplementary information accompanies this paper at http://www.nature.com/srep

Competing financial interests: The authors declare no competing financial interests.

How to cite this article: Luo, C. et al. Ecological impact of a secondary bacterial symbiont on the clones of Sitobion avenae (Fabricius) (Hemiptera: Aphididae). Sci. Rep. 7, 40754; doi: 10.1038/srep40754 (2017).

Publisher's note: Springer Nature remains neutral with regard to jurisdictional claims in published maps and institutional affiliations. 
(c) (i) This work is licensed under a Creative Commons Attribution 4.0 International License. The images or other third party material in this article are included in the article's Creative Commons license, unless indicated otherwise in the credit line; if the material is not included under the Creative Commons license, users will need to obtain permission from the license holder to reproduce the material. To view a copy of this license, visit http://creativecommons.org/licenses/by/4.0/

(C) The Author(s) 2017 